DOI: 10.12731/2658-6649-2020-12-4-25-37

УДК 616-072.7

\title{
ЦЕРЕБРАЛЬНАЯ МИКРОЭМБОЛИЯ \\ У ПАЦИЕНТОВ С ЗАБОЛЕВНИЯМИ СЕРДЕЧНО- СОСУДИСТОЙ СИСТЕМЫ, НАХОДЯЩИХСЯ НА РЕАБИЛИТАЦИИ ПОСЛЕ НАРУШЕНИЯ МОЗГОВОГО КРОВООБРАЩЕНИЯ, ТЯЖЕЛЫХ ПОВРЕЖДЕНИЙ ГОЛОВНОГО МОЗГА
}

\author{
Скворцов А.Е., Яковлев А.А., Яковлева А.В., Щелкунова И.Г.
}

В статье приведен анализ результатов исследования частоты выявления микроэмболии в средних мозговых артериях у пациентов с заболеваниями сердечно-сосудистой системы: стенозирующими атеросклерозом иеребральных артерий, фибриллящией предсердий, проходящих лечение и реабилитацию после нарушения мозгового кровообращения, тяжёльхх повреждений головного мозга вследствие сосудистых заболеваний (аневризма сосудов головного мозга, ишемический и геморрагический инсульт), черепно-мозговой травмы, опухолей головного мозга, в том числе, после нейрохирургических вмешательств в сравнении с практически здоровыми людьми.

Всем обследованным был выполнен транскраниальный допплерографический мониторинг интракраниальных артерий. Детекция микроэмболов осуществлялась в ходе двухстороннего транскраниального допплеровского мониторирования потоков в средних мозговых артериях. K naтологическим относили высокоэнергетические сигналы с относительным повышением мощности более $20 \mathrm{~dB} / \mathrm{ms}$, во время которых допплеровская мощность возрастала как минимум на $5 \mathrm{~dB}$ и длилась не менее $4 \mathrm{~ms}$. Для оценки ультразвуковых характеристик атеросклеротических блятек с помощью дуплексного сканирования у пациентов со стенозирующим атеросклерозом брахиоцефальных артерий использовали классификацию Gray-Weale A.C с соавт. Выявление и оценка атеросклеротических блятек осуществлялась в ходе плановых исследований магистральных артерий головы линейным датчиком ультразвукового прибора DC-8, Mindray.

В ходе исследования признаки микроэмболии были зарегистрированы во всех отобранныл группах. Реже всего они выявлялись в контрольной 
группе у практически здоровых лии, что объясняется отсутствием явных предпосылок к образованию микроэмболов. В группе паџиентов с постоянной формой фибрилляцией предсердий количество случаев выявления микроэмболии не превысила половины обследованных. В группах сравнения у пациентов со стенозирующим атеросклерозом отмечается наибольшая частота выявления микроэмболии у пациентов с гетерогенными атеросклеротическими блямками. И это совпадает с распределением среднего количества микроэмболических сигналов.

Ключевые слова: ичеребральная микроэмболия; микроэмболический сигнал; ультразвуковая допплерография; иеребральные артерии; коморбидная патология.

\section{CEREBRAL MICROEMBOLISM IN PATIENTS WITH DISEASES OF THE CARDIOVASCULAR SYSTEM, WHO ARE IN REHABILITATION AFTER A VIOLATION OF CEREBRAL CIRCULATION, SEVERE BRAIN INJURIES}

\section{Skvorcov A.E., Yakovlev A.A., Yakovleva A.V., Shhelkunova I.G.}

The article presents the analysis of the study results the detection rate of microemboli in middle cerebral arteries of patients with cardiovascular disease: stenosing atherosclerosis of cerebral arteries, atrial fibrillation, a condition after implantation of an artificial heart valve, treatment and rehabilitation after cerebrovascular accident, severe damage to the brain due to vascular diseases (aneurysm of cerebral vessels, ischemic and hemorrhagic stroke), traumatic brain injury, brain tumors, including, after neurosurgical interventions in comparison with practically healthy people.

Transcranial Doppler monitoring of intracranial arteries was performed for all the examined patients. Microembolism detection was performed during two-way transcranial Doppler monitoring of flows in the middle cerebral arteries. Pathological signals included high-energy signals with a relative power increase of more than $20 \mathrm{~dB} / \mathrm{ms}$, during which the Doppler power increased by at least $5 \mathrm{~dB}$ and lasted for at least $4 \mathrm{~ms}$. To evaluate the ultrasound characteristics of atherosclerotic plaques using duplex scanning in patients with stenotic atherosclerosis of the brachiocephalic arteries, the classification A. Gray-Weale et al. The detection and evaluation of atherosclerotic plaques was carried out 
during routine studies of the main arteries of the head using a linear sensor of the DC-8 ultrasound device, Mindray.

During the study, signs of microembolism were registered in all the selected groups. They were less often detected in the control group in practically healthy individuals, which is explained by the lack of clear prerequisites for the formation of microemboluses. In the group of patients with a permanent form of atrial fibrillation, the number of cases of microembolism detection did not exceed half of the examined patients. In comparison groups, patients with stenotic atherosclerosis have the highest incidence of microembolism in patients with heterogeneous atherosclerotic plaques. And coincides with the distribution of the average number of microembolic signals.

Keywords: cerebral microembolism; microembolic signal; ultrasound dopplerography; cerebral arteries; comorbid pathology.

\section{Введение}

Важной составляющей комплексного исследования церебральной гемодинамики является детекция церебральной микроэмболии. Ультразвуковая допплерография позволяет осуществлять прямую детекцию эмболического материала, движущегося по сосудам головного мозга [1, $2,3]$. При прохождении микроэмбола через исследуемый участок сосуда регистрируется микроэмболический сигнал. Под микроэмболическим сигналом следует понимать допплеровский сигнал, обладающий следующими характеристиками: транзиторностью (его длительность не должна превышать 300 мс), высокой амплитудой (амплитуда микроэмболического сигнала как минимум на 3 дБ выше фонового сигнала), внутриспектральным положением, ограниченным изолинией. Микроэмболический сигнал следует отличать от мультиформных артефактных сигналов. С этой целью используются различные современные технологии, позволяющие значительно снизить ошибки при регистрации и фильтровании полученных сигналов [4-8].

Опыт проведенных исследований показывает неоднородность состава микроэмболических сигналов. Их можно разделить на газовые (воздушные), материальные (солидные). Состав солидных микроэмболов может включать микропузырьки газа, агрегаты тромбоцитов, фрагменты тромбов, вегетаций и опухоли, атероматозные массы, липидный компонент, кальцинаты, синтетические материалы. Различный состав микроэболов определяется полиэтиологичностью состояний, сопровождающихся их образованием [9-11]. 
Спектр сердечно-сосудистой патологии, ассоциированной с риском церебральной и системной эмболии, разнообразен. Многие работы посвящены изучению микроэмболизации у пациентов с сердечно-сосудистой патологией, а именно с искусственными клапанами сердца и нарушениями ритма сердца. Часть исследователей придерживаются теории о газовой природе микроэмболов [12-14]. Некоторые отрицают роль микроэмболов в развитии церебральных ишемических событий, и вместе с тем показывают связь длительной микроэмболизации с развитием когнитивных нарушений [15-16]. Есть работы, в которых наряду с признанием возможности образования микропузырьков, авторы указывают на очевидное присутствие материальных микроэмболов которые обладают клинической значимостью, предопределяя развитие клинической манифестации $[9,11]$.

Нарушения ритма сердца являются одним из самым частым источников микроэмболии, приводящим к формированию ишемического некроза в веществе мозга и частота эта увеличивается по мере старения населения. Фибрилляция предсердий является самой частой причиной кардиоэмболческих инсультов и считается наиболее распространенным нарушением ритма сердца [17]. У людей старше 60 лет она встречается у 6\%, а до 60 лет - у 1\% [18]. У пациентов с фибрилляцией предсердий тромбы обычно формируются в ушке левого предсердия и стоком крови попадают в сосуды головного мозга. Кроме того, нарушения ритма сердца усугубляют церебральную гемодинамику, влияя на течение инсульта и прогноз заболевания.

Прогноз всегда хуже у пациентов с коморбидной патологией. Было установлено, что при наличии стенозирующего атеросклероза церебральных артерий частота детекции микроэмболов и их количество выше в бассейне сосуда со стенозом [19-21]. Кроме того, частота микроэмболии и количество микроэмболических сигналов прямо коррелирует со степенью стеноз и целостностью атеросклеротической бляшки [22].

Главным в допплеровской детекции церебральной эмболии было и остается клиническое значение обнаруженных микроэмболических сигналов у пациентов разных групп.

\section{Цель настоящего исследования}

В своей работе мы проводили анализ частоты возникновения микроэмболии у пациентов с заболеваниями сердечно-сосудистой системы, проходящих лечение и реабилитацию после нарушения мозгового кровообращения, тяжёлых повреждений головного мозга вследствие сосуди- 
стых заболеваний (аневризма сосудов головного мозга, ишемический и геморрагический инсульт), черепно-мозговой травмы, опухолей головного мозга, в том числе, после нейрохирургических вмешательств в сравнении с практически здоровыми людьми.

\section{Характеристика клинического материала}

В исследование были включены результаты обследования 100 человек, большая часть из которых -80 человек проходили лечение в Федеральном научно-клиническом центре реаниматологии и реабилитологии (ФНКЦ PР), остальные 20 человек это практически здоровые по результатам ежегодного медицинского обследования добровольцы, которые были включены в качестве контрольной группы.

Средний возраст первой группы составил 49 лет, второй группы - 46 лет. Как видно из таблицы 1 в первую группы были включены пациенты с признаками стенозирующего атеросклероза брахиоцефальных артерий 40 человек, с постоянной формой фибрилляции предсердий - 40 человек. Двое пациентов с постоянной формой фибрилляции предсердий имели искусственные клапаны сердца. Всем обследованным был выполнен транскраниальный допплерографический мониторинг интракраниальных артерий. Детекция микроэмболов осуществлялась в ходе двухстороннего транскраниального допплеровского мониторирования потоков в средних мозговых артериях.

Таблииа 1.

Распределение исследуемых по группам

\begin{tabular}{|c|l|c|}
\hline \multirow{2}{*}{ ГРУППЫ } & \multicolumn{1}{|c|}{ НОЗОЛОГИИ } & $\begin{array}{c}\text { КОЛИЧЕСТВО } \\
\text { ЧЕЛОВЕК }\end{array}$ \\
\hline \multirow{2}{*}{1} & Стенозирующей атеросклероз & 40 \\
\cline { 2 - 3 } & $\begin{array}{l}\text { Постоянная форма фибрилляции } \\
\text { предсердий }\end{array}$ & 40 \\
\hline 2 & Практически здоровые добровольцы & 20 \\
\hline
\end{tabular}

Для зарегистрированных микроэмболических сигналов определяли мощность (dB), частоту (Гц) и длительность (ms). К патологическим относиливысокоэнергетические сигналы с относительным повышением мощности более $20 \mathrm{~dB} / \mathrm{ms}$, во время которых допплеровская мощность возрастала как минимум на $5 \mathrm{~dB}$ и длилась не менее $4 \mathrm{~ms}$.

Для оценки ультразвуковых характеристик атеросклеротических бляшек с помощью дуплексного сканирования у пациентов со стенозирующим 
атеросклерозом брахиоцефальных артерий использовали классификацию A. Gray-Wealeetal.: 1-й тип - однородные гипоэхогенные; 2-й тип - однородные гиперэхогенные; 3-й тип - неоднородные с преобладанием гипоэхогенного компонента; 4-й тип - неоднородные с преобладанием гиперэхогенного компонента. Выявление и оценка атеросклеротических бляшек осуществлялась в ходе плановых исследований магистральных артерий головы линейным датчиком ультразвукового прибора DC-8, Mindray.

\section{Результаты}

В группе практически здоровых добровольцев церебральная микроэмболия была выявлена у 3 пациентов из 20 (15\%). Число зарегистрированных микроэмболических сигналов в среднем составило 1: от 1 до 2 сигналов за 60 мин.

В группе пациентов с постоянной формой фибрилляцией предсердий церебральную микроэмболию выявляли у 19 пациентов из 40 (47,5\%). Число зарегистрированных микроэмболических сигналов в среднем составило 9: от 1 до 22 сигналов за 60 мин. У обоих пациентов с имплантированным искусственным клапаном сердца были выявлены признаки церебральной микроэмболии. Число зарегистрированных микроэмболических сигналов в среднем составило 4: от 2 до 6 сигналов за 60 мин.

В группе со стенозирующим атеросклерозом брахицефальных артерий пациенты были распределены на четыре группы с учетом преобладающего типа атеросклеротических бляшек (АСБ). Однородные гипоэхогенные АСБ (1-й тип) были обнаружены у 5 пациентов, однородные гиперэхогенные (2-й тип) у 2 пациентов неоднородные с преобладанием гипоэхогенного компонента (3-й тип) - у 11 пациентов; неоднородные с преобладанием гиперэхогенного компонента (4-й тип) - у 22 пациентов.

В группе больных с неоднородными гипоэхогенными АСБ (3-й тип) церебральную микроэмболию выявляли у 10 пациентов из 11 (91\%). Число зарегистрированных микроэмболических сигналов в среднем составило 18: от 1 до 33 сигналовза 60 мин. У пациентов с неоднородными гиперэхогенными АСБ (4-й тип) церебральную микроэмболию выявляли у 9 из 22 больных (41\%), среднее количество микроэмболических сигналов составило 5: от 1 до 10 сигналов за 60 мин. У больных с однородными гипоэхогенными бляшками (1-й тип) микроэмболические сигналы зарегистрировали у 4 из 5 пациентов (80\%), среднее количество микроэмболических сигналов составило 3: от 1 до 6 сигналов за 60 мин, а в группе больных с однородными гиперэхогенными бляшками (2-й тип) к которой 
были отнесены два пациента, три микроэмболических сигнала за 60 минут были выявлены у одного пациента.

Таким образом, в ходе исследования признаки микроэмболии были зарегистрированы во всех сформированных группах. Реже всего они выявлялись в контрольной группе у практически здоровых лиц, что объясняется отсутствием явных предпосылок к образованию микроэмболов, что не позволяет исключить наличие не выявленных заболеваний у 3 человек из этой группы. В группе пациентов с постоянной формой фибрилляции предсердий количество случаев выявления микроэмболии не превысила половины обследованных. Выявление микроэмболии у обоих пациентов с искусственный клапан сердца указывает на существенно возрастающую вероятность микроэмболии в случае сочетанной патологии сердца. В группах сравнения у пациентов со стенозирующим атеросклерозом отмечается высокая частота выявления микроэмболии у пациентов с гетерогенными атеросклеротическими бляшками: 3-й тип составляет 91\%, 4-ый тип 41\% соответственно. И это совпадает с распределением среднего количества микроэмболических сигналов.

\section{Обсуждение}

Разнообразный спектр сердечно-сосудистой патологии, ассоциированной с риском церебральной и системной эмболии у различных категорий пациентов требует внимательного изучения. Широкая распространенность атеросклеротического поражения сосудов, нарушений ритма сердца у пациентов, проходящих лечение и реабилитацию после нарушения мозгового кровообращения, тяжёлых повреждений головного мозга вследствие сосудистых заболеваний (аневризма сосудов головного мозга, ишемический и геморрагический инсульт), черепно-мозговой травмы, опухолей головного мозга, в том числе, после нейрохирургических вмешательств требует проведения анализа частоты возникновения микроэмболии.

Полученные данные подтвердили факт частого возникновения микроэмболии как у пациентов с атеросклерозом сонных артерий, так и у пациентов с ишемической болезнью сердца, фибрилляцией предсердий. Результаты сопоставимы с данными, приведёнными в работе Przemyslaw Puz и соавт. данными, где было продемонстрировано, что микроэмболические сигналы регистрируются достоверно чаще у пациентов с гипоэхогенной структурой атеросклеротических бляшек [23]. Также как и в работе D Georgiadisu coавт. [24] частота выявления микроэмболии у пациентов с трепетанием предсердия не превышала $50 \%$, в то время как у пациентов 
с сочетанной патологией сердца вероятность микроэмболии была существенно выше. Проведенное исследование 80 пациентов и анализ случаев эмболодетекции подтвердило широкую распространенность микроэмболии среди контингента, проходящего лечение и реабилитацию в центре, не выявило строгой специфичности для выбранной патологии.

\section{Выводы}

Полученные данные подтверждают полиэтиологичность природы возникновения микроэболов, указывают на повышение риска развития микроэмболии сосудов голоного мозга в группах пациентов с такими заболеваниями сердечно-сосудистой системы как стенозирующий атеросклероз магистральных артерий, нарушения ритма сердца.

\section{Список литературы}

1. Kumral E., Balkir K., Yagdi T., Kara E., Evyapan D., Bilkay O. Microembolic signals in patients undergoing coronary artery bypass grafting // Tex. Heart Inst. J. 2001. V.28. P. 16-20.

2. Spencer M.P., Thomas G., Hicholls S.C., Sauvage L.R. Detection of middle cerebral artery emboli during carotid endarterectomy using transcranial Doppler ultrasonography // Neurology. 1990. V. 21. P. 415-423.

3. Tong D.C., Albers G.W. Microembolic signals in patients undergoing coronary bypass grafting // Stroke. 1995. V. 26. P. 1588-1592.

4. Molloy J., Markus H.S. Multigated Doppler ultrasound in the detection of emboli in a flow model and embolic signals in patients//Stroke. 1996. V. 27. P. 1548-1552.

5. Smith J.L., Evans D.H., Fan L., Bell P.R., Naylor A.R. Differentiation between emboli and artefacts using dual-gated transcranial Doppler ultrasound // Ultrasound Med. Biol.1996. V.22. P. 1031-1036.

6. Moehring M.A. Microembolus tracking with power M-mode transcranial Doppler ultrasound and simultaneous single gate spectrogram II // Cerebrovasc. Dis. 2000. V. 10. Suppl. 1.P.2.

7. Fan L., Evans D.H., Naylor A.R. Automated emboli identification Detection using a combination of time and frequency domain information // Cerebrovasc. Dis. 2000. V. 10. Suppl. 1. P. 1.

8. Uhlmann F., Schulte-Mattler W.J., Georgladis D. Postembolic spectral patterns of microembolic signals // Cerebrovasc. Dis. 2000. V. 10. Suppl. 1. P. 3.

9. Georgiadis D. Intracranial microembolic signals in patients with artificial heart valves: Drowning in numbers. / D. Georgiadis, A. Lindner, S. Zeirz // Eur.J.Med. Res. 1998. Vol. 3. P. 99-102. 
10. Климов И.А. Неврологические осложнения инфекционного эндокардита: особенности течения и диагностики: автореф. дис. ... канд. мед. наук. СПб, $2001.25 \mathrm{c}$.

11. Warlow C.P. Stroke: A Practical Guide to Management / C.P. Warlow, M.S. Dennis, J. van Gijn, A. Hankey. Oxford, 1996. Vol. 6. P. 183-190.

12. Ggeorgiadis D., Baumgart R.W., Karatschai F. Further evidence of gaseous embolic material in patients with artificial heart valves // J. Thorac. Cerebrovasc. Surg. 1998. V. 115 P. 808-810.

13. Georgiadis D., Lindner A., Zierz S. Intracranial microembolic signals in patients with artificial heart valves: Drowning in numbers // Europ. J. Med.1998. V. 3. P. 99-102.

14. Georgiadis D. Microembolism in prosthetic valve patients // Stroke. 1998. V. 29. P. 2237.

15. Deklunder G., Prat A., Lecroart J.L., Roussel M., Dauzat M. Can cerebrovascular microemboli induce cognitive impairment in patients with prosthetic heart valves? //Europ. J Ultrasound. 1998.V.7. P. 47-51.

16. Deklunder G., Roussel M., Lecroart J.L., Prat A., Gautier C. Microemboli in cerebral circulation and alteration of cognitive abilities in patients with mechanical prosthetic heart valves // Stroke. 1998. V. 29. P. 1821-1826.

17. Диагностика и лечение фибрилляции предсердий / Рекомендации РКО, BНОА, АССХ. М.: «Байер». 2012. 65 с.

18. Пономарёва Е.Н. Кардиогенные инфаркты мозга // Журнал «Медицинские новости». 2005. №10. С.4-8.

19. Babikian V.L., Hyde C., Pochay V., Winter M.R. Clinical correlates of high-intensity transient signals detected on transcranial Doppler sonography in patients with cerebrovascular disease// Stroke. 1994. V. 25. P. 1570-1573.

20. Markus H.S., Thomson N.D., Brown M.M. Asymptomatic cerebral embolic signals in symptomatic and asymptomatic carotid artery disease // Brain. 1995. V. 118. P. 1005-1011.

21. Orlandi G., Parenti G., Bertolucci A., Murri L. Silent cerebral microembolism in asymptomatic and symptomatic carotid artery stenosis of low and high degree // Eur. Neurol. 1997;38(1):39-43.

22. Droste D.W., Dittrich R., Kemeny V. et al. Prevalence and frequency of microembolic signals in 105 patients with extracranial carotid artery occlusive disease // J Neurol. Neurosurg. Psychiatry. 1999. V. 67. P. 525-528.

23. Puz P., Lasek-Bal A., Urbanek T., Kazibutowska Z. Assessment of cerebral embolism and vascular reserve parameters in patients with carotid artery stenosis // Neurol. Neurochir. Pol., 50 (2016). P. 356-362. 
24. Georgiadis D., Lindner A., Manz M., Sonntag M., Zunker P., Zerkowski H.R., Borggrefe M. Intracranial Microembolic Signals in 500 Patients With Potential Cardiac or Carotid Embolic Source and in Normal Controls // Stroke. 1997. Jun;28(6):1203-7.

\section{References}

1. Kumral E., Balkir K., Yagdi T., Kara E., Evyapan D., Bilkay O. Microembolic signals in patients undergoing coronary artery bypass grafting. Tex. Heart Inst. J. 2001. V.28. P.16-20.

2. Spencer M.P., Thomas G., Hicholls S.C., Sauvage L.R. Detection of middle cerebral artery emboli during carotid endarterectomy using transcranial Doppler ultrasonography. Neurology. 1990. V. 21. P. 415-423.

3. Tong D.C., Albers G.W. Microembolic signals in patients undergoing coronary bypass grafting. Stroke. 1995. V. 26. P. 1588-1592.

4. Molloy J., Markus H.S. Multigated Doppler ultrasound in the detection of emboli in a flow model and embolic signals in patients. Stroke. 1996. V. 27. P. 1548-1552.

5. Smith J.L., Evans D.H., Fan L., Bell P.R., Naylor A.R. Differentiation between emboli and artefacts using dual-gated transcranial Doppler ultrasound. Ultrasound Med. Biol.1996. V.22. P. 1031-1036.

6. Moehring M.A. Microembolus tracking with power M-mode transcranial Doppler ultrasound and simultaneous single gate spectrogram II. Cerebrovasc. Dis. 2000. V. 10. Suppl. 1. P.2.

7. Fan L., Evans D.H., Naylor A.R. Automated emboli identification Detection using a combination of time and frequency domain information. Cerebrovasc. Dis. 2000. V. 10. Suppl. 1. P. 1.

8. Uhlmann F., Schulte-Mattler W.J., Georgladis D. Postembolic spectral patterns of microembolic signals. Cerebrovasc. Dis. 2000. V. 10. Suppl. 1. P. 3.

9. Georgiadis D., Lindner A., Zeirz S. Intracranial microembolic signals in patients with artificial heart valves: Drowning in numbers. Eur.J.Med.Res. 1998. Vol. 3. P. 99-102.

10. Klimov I.A. Nevrologicheskie oslozhnenija infekcionnogo jendokardita: osobennosti techenija i diagnostiki [Neurological complications of infectious endocarditis: features of the course and diagnosis]. Cand. philos. sci. diss. Abstr. St. Petersburg, 2001, 25 p.

11. Warlow C.P. Stroke: A Practical Guide to Management / C.P. Warlow, M.S. Dennis, J. van Gijn, A. Hankey. Oxford, 1996. Vol. 6. P. 183-190.

12. Ggeorgiadis D., Baumgart R.W., Karatschai F. Further evidence of gaseous embolic material in patients with artificial heart valves. J. Thorac. Cerebrovasc. Surg. 1998. V. 115 P. 808-810. 
13. Georgiadis D., Lindner A., Zierz S. Intracranial microembolic signals in patients with artificial heart valves: Drowning in numbers. Europ. J. Med.1998. V. 3. P. 99-102.

14. Georgiadis D. Microembolism in prosthetic valve patients. Stroke. 1998. V. 29. P. 2237.

15. Deklunder G., Prat A, Lecroart J.L., Roussel M., Dauzat M. Can cerebrovascular microemboli induce cognitive impairment in patients with prosthetic heart valves? Europ.J Ultrasound. 1998. V.7. P. 47-51.

16. Deklunder G., Roussel M., Lecroart J.L., Prat A., Gautier C. Microemboli in cerebral circulation and alteration of cognitive abilities in patients with mechanical prosthetic heart valves. Stroke. 1998. V. 29. P. 1821-1826.

17. Diagnostika i lechenie fibrilljacii predserdij. Rekomendacii Rossijskogo Kardiologicheskogo obshhestva, Obshhestva specialistov po klinicheskoj jelektrofiziologii, Aritmologii i jelektrokardiostimuljacii i Associacii serdechno-sosudistyh hirurgov Rossii [Diagnosis and treatment of atrial fibrillation. Recommendations of the Russian Cardiological Society, the Society of Specialists in Clinical Electrophysiology, Arrhythmology and Electrocardiostimulation, and the Association of Cardiovascular Surgeons of Russia]. Moscow: Bayer, 2012. 65 p.

18. Ponomarjova E.N. Kardiogennye infarkty mozga [Cardiogenic cerebral infarction]. Medicinskie novosti [Medical News Magazine] 2005. №10. pp. 4-8.

19. Babikian V.L., Hyde C., Pochay V., Winter M.R. Clinical correlates of high-intensity transient signals detected on transcranial Doppler sonography in patients with cerebrovascular disease. Stroke. 1994. V. 25. P. 1570-1573.

20. Markus H.S., Thomson N.D., Brown M.M. Asymptomatic cerebral embolic signals in symptomatic and asymptomatic carotid artery disease. Brain. 1995. V. 118. P. 1005-1011.

21. Orlandi G., Parenti G., Bertolucci A., Murri L. Silent cerebral microembolism in asymptomatic and symptomatic carotid artery stenosis of low and high degree. Eur. Neurol. 1997;38(1):39-43.

22. Droste D.W., Dittrich R., Kemeny V. et al. Prevalence and frequency of microembolic signals in 105 patients with extracranial carotid artery occlusive disease. J Neurol. Neurosurg. Psychiatry. 1999. V. 67. P. 525-528.

23. Puz P., Lasek-Bal A., Urbanek T., Kazibutowska Z. Assessment of cerebral embolism and vascular reserve parameters in patients with carotid artery stenosis. Neurol. Neurochir. Pol., 50 (2016), pp. 356-362.

24. Georgiadis D., Lindner A., Manz M., Sonntag M., Zunker P., Zerkowski H.R., Borggrefe M. Intracranial Microembolic Signals in 500 Patients With Potential Cardiac or Carotid Embolic Source and in Normal Controls. Stroke. 1997 Jun;28(6):1203-7. 


\section{ДАННЫЕ ОБ АВТОРАХ}

Скворцов Артем Евгеньевич, заведующий отделением функциональной диагностики

Федеральное государственное бюджетное научное учреждение «Федеральный научно-клинический центр реаниматологии и реабилиталогии»

ул. Петровка, 25, стр. 2, 107031, г. Москва, Российская Федераичия

Яковлев Алексей Александрович, заместитель руководителя НИИ реабилитологии

Федеральное государственное бюджетное научное учреждение «Федеральный научно-клинический иентр реаниматологии и реабилиталогии»

ул. Петровка, 25, стр. 2, 107031, г. Москва, Российская Федераиุия

Яковлева Александра Витальевна, младший научный сотрудник лаборатории клинического питания и метаболизма

Федеральное государственное бюджетное научное учреждение «Федеральный научно-клинический центр реаниматологии и реабилиталогии»

ул. Петровка, 25, стр. 2, 107031, г. Москва, Российская Федераџия ayakovleva@fnkcrr.ru

Щелкунова Инесса Геннадиевна, заместитель директора по организации медицинской деятельности

Федеральное государственное бюджетное научное учреждение «Федеральный научно-клинический центр реаниматологии и реабилиталогии»

ул. Петровка, 25, стр. 2, 107031, г. Москва, Российская Федераџия

DATA ABOUT THE AUTHORS

Skvorcov Artem Evgenevich, Head of the Department of Functional Diagnostics

Federal Research and Clinical Center of Intensive Care Medicine and Rehabilitology

25, build. 2, Petrovka Str., 107031, Moscow, Russian Federation 
Yakovlev Aleksey Aleksandrovich, Deputy Head of the Research Institute of Rehabilitation

Federal Research and Clinical Center of Intensive Care Medicine and Rehabilitology

25, build. 2, Petrovka Str., 107031, Moscow, Russian Federation

Yakovleva Aleksandra Vitalevna, Junior Researcher, Laboratory of Clinical Nutrition and Metabolism

Federal Research and Clinical Center of Intensive Care Medicine and Rehabilitology

25, build. 2, Petrovka Str., 107031, Moscow, Russian Federation ayakovleva@fnkcrr.ru

Shhelkunova Inessa Gennadievna, Deputy Director for Organization of Medical Activities

Federal Research and Clinical Center of Intensive Care Medicine and Rehabilitology

25, build. 2, Petrovka Str., 107031, Moscow, Russian Federation 